\title{
A new standard nomenclature for proteins related to Apx and Shroom
}

\author{
Olivier Hagens ${ }^{1}$, Andrea Ballabio ${ }^{2}$, Vera Kalscheuer ${ }^{1}$, Jean- \\ Pierre Kraehenbuhl3 , M Vittoria Schiaffino ${ }^{4}$, Peter Smith ${ }^{5}$, Olivier Staub 6 , \\ Jeff Hildebrand ${ }^{7}$ and John B Wallingford*8
}

\begin{abstract}
Address: ${ }^{1}$ Dept. of Human Molecular Genetics, Max Planck Institute for Molecular Genetics, Berlin, Germany, ${ }^{2}$ Telethon Institute of Genetics and Medicine, Naples, Italy, ${ }^{3}$ Swiss Institute for Experimental Cancer Research and the Institute of Biochemistry, University of Lausanne, Lausanne, Switzerland, ${ }^{4}$ Dept. of Biotechnology, San Raffaele Scientific Institute, Milan, Italy, ${ }^{5}$ Dept. of Physiology and Biophysics, University of Alabama at Birmingham, Birmingham, AL, USA, ${ }^{6}$ Dept. of Pharmacology \& Toxicology, University of Lausanne, Lausanne, Switzerland, ${ }^{7}$ Dept. of Biological Sciences, University of Pittsburgh, Pittsburgh, PA, USA and ${ }^{8}$ Dept. of Molecular Cell and Developmental Biology \& Institute for Cellular and Molecular Biology, University of Texas, Austin, TX, USA

Email: Olivier Hagens - hagens@molgen.mpg.de; Andrea Ballabio - ballabio@tigem.it; Vera Kalscheuer - kalscheu@molgen.mpg.de; JeanPierre Kraehenbuhl - Jean-Pierre.Kraehenbuhl@isrec.ch; M Vittoria Schiaffino - schiaffino.mariavittoria@hsr.it;

Peter Smith - Smith@PHYSIOLOGY.UAB.EDU; Olivier Staub - olivier.staub@unil.ch; Jeff Hildebrand - jeffh+@pitt.edu;

John B Wallingford* - wallingford@mail.utexas.edu

* Corresponding author
\end{abstract}

Published: 14 April 2006

BMC Cell Biology 2006, 7:18 doi:10.1/86/147|-2121-7-18

This article is available from: http://www.biomedcentral.com//47|-2/2I/7//8

(C) 2006 Hagens et al; licensee BioMed Central Ltd.

This is an Open Access article distributed under the terms of the Creative Commons Attribution License (http://creativecommons.org/licenses/by/2.0), which permits unrestricted use, distribution, and reproduction in any medium, provided the original work is properly cited.
Received: 29 March 2006

Accepted: 14 April 2006

\begin{abstract}
Shroom is a recently-described regulator of cell shape changes in the developing nervous system. This protein is a member of a small family of related proteins that are defined by sequence similarity and in most cases by some link to the actin cytoskeleton. At present these proteins are named Shroom, APX, APXL, and KIAAI202. In light of the growing interest in this family of proteins, we propose here a new standard nomenclature.
\end{abstract}

In 1992, the primary structure of an apical protein in Xenopus (Apx) was described [1]. Since then, three related proteins have been characterized, namely the human proteins APXL (apical protein Xenopus-like) [2] and KIAA1202 [3] and mouse Shroom [4], named after the mouse mutant phenotype. We now know that the Apx protein of Xenopus is not in fact the orthologue of human APXL. Instead, the protein previously called human APXL2 is the likely homologue of frog Apx, while human APXL is the likely homologue of a Xenopus APXL. In this letter, we report a new standardized nomenclature to eliminate the confusing present naming situation for these proteins (Table 1 ).
From global multiple alignments of genomic sequences, it is clear that these proteins are not simply encoded by homologous genes. There are in fact four different proteins in this family, showing similarity in their domains (Table 2), which include a PDZ and two Apx/Shrm domains (ASD1 and ASD2) and putative EVH1 and PDZ binding sites [4]. It should be noted however that Apx lacks the PDZ domain and the EVH1 binding site, APXL lacks a PDZ binding site and KIAA1202 does not contain an obvious ASD1 domain. Therefore, the ASD2 domain seems to be the common denominator among family members. 
Table I: New nomenclature for Shroom-related proteins

\begin{tabular}{|c|c|c|}
\hline $\begin{array}{l}\text { GenBank Accession } \\
\text { Number }\end{array}$ & Previous name & New name \\
\hline CAA78718 & X. laevis Apx & xShroom I \\
\hline NP 597713 & H. sapiens APXL2 & hShroom I \\
\hline$\overline{\text { CAA58534 }}$ & H. sapiens APXL & hShroom2 \\
\hline$\underline{\mathrm{ABD} 19518}$ & M. musculus Apxl & mShroom2 \\
\hline AAFI3269 & M. musculus ShroomL & mShroom3a \\
\hline AAFI3270 & M. musculus ShroomS & mShroom3b \\
\hline NP 065910 & H. sapiens Shroom & hShroom3 \\
\hline ABD59319 & $X$. laevis Shroom-like & xShroom3 \\
\hline NP 065768 & H. sapiens KIAAI 202 & hShroom4a \\
\hline AAK95579 & H. sapiens SHAP-A & hShroom4b \\
\hline DQ435686 & M. musculus KIAAI 202 & mShroom4 \\
\hline ABA81834 & D. melanogaster Shroom & dmShroom \\
\hline$\overline{\text { EAAI } 2598}$ & A. gambiae Shroom & agShroom \\
\hline XP 392427 & A. mellifera Shroom & amShroom \\
\hline XP 783573 & S. purpuratus Shroom & spShroom \\
\hline
\end{tabular}

Bioinformatics-based searches identified Shroom-related proteins in all chordates examined. In addition, insect genomes, including Drosophila melanogaster, Anopheles gambiae and Apis mellifera, encode a partially related protein containing an ASD2 domain (Table 1). Finally, BLAST searches of the deposited sequences from invertebrate genome projects identify what may be considered Shroom orthologues in both Ciona intestinalis (data not shown) and Strongylocentrotus purpuratus (Table 1). Based on the putative open reading frames and genomic organization, these predicted proteins contain, at least, the $\mathrm{N}$ terminal PDZ domain and the C-terminally positioned ASD2 motif.

To clarify future studies, we propose a unifying nomenclature, emphasizing the relatedness of those proteins (Table 1 ). We feel that while the founding member is Apx, this name is undesirable as a root for naming this family because it requires that 'Xenopus' would appear in protein names from all species. Instead, we propose that the new nomenclature be based upon the name 'Shroom' as this is now the most thoroughly studied member of the family [4-6]. An Arabic number following 'Shroom' would distinguish between the different proteins. A lower-case letter would distinguish between different protein products encoded by the same locus generated by alternative mRNA processing. According to these rules, we suggest the re-naming presented in Table 1.

Several papers suggest that these related proteins play diverse and important roles in the development of the nervous system and other tissues [2-8]. Future studies will be required to show if sequence similarity among Shroom protein family members is mirrored by conservation of their cellular and molecular function.

\section{References}

I. Staub O, Verrey F, Kleyman TR, Benos DJ, Rossier BC, Kraehenbuhl JP: Primary structure of an apical protein from Xenopus laevis that participates in amiloride-sensitive sodium channel activity. J Cell Biol 1992, I I 9(6): |497-I506.

2. Schiaffino MV, Bassi MT, Rugarli El, Renieri A, Galli L, Ballabio A Cloning of a human homologue of the Xenopus laevis APX gene from the ocular albinism type I critical region. Hum $\mathrm{Mol}$ Genet 1995, 4(3):373-382.

3. Hagens O, Dubos A, Abidi F, Barbi G, Van Zutven L, Hoeltzenbein M, Tommerup N, Moraine C, Fryns JP, Chelly J, van Bokhoven H, Gecz J, Dollfus H, Ropers HH, Schwartz CE, de Cassia Stocco Dos Santos R, Kalscheuer V, Hanauer A: Disruptions of the novel KIAAI 202 gene are associated with $\mathbf{X}$-linked mental retardation. Hum Genet 2005: I- I3.

4. Hildebrand JD, Soriano P: Shroom, a PDZ domain-containing actin-binding protein, is required for neural tube morphogenesis in mice. Cell 1999, 99(5):485-497.

5. Haigo SL, Hildebrand JD, Harland RM, Wallingford JB: Shroom induces apical constriction and is required for hingepoint formation during neural tube closure. Curr Biol 2003, I3(24):2 1 25-2। 37.

6. Hildebrand JD: Shroom regulates epithelial cell shape via the apical positioning of an actomyosin network. J Cell Sci 2005, I | 8:519|-5203.

7. Prat AG, Holtzman EJ, Brown D, Cunningham CC, Reisin IL, Kleyman TR, McLaughlin M, Jackson GRJ, Lydon J, Cantiello HF: Renal epithelial protein (Apx) is an actin cytoskeleton-regulated $\mathrm{Na}+$ channel. J Biol Chem I996, 27 I(30): I8045-18053.

8. Zuckerman JB, Chen X, Jacobs JD, Hu B, Kleyman TR, Smith PR: Association of the epithelial sodium channel with Apx and alpha-spectrin in A6 renal epithelial cells. J Biol Chem 1999, 274(33):23286-23295.

Table 2: Sequence identity matrix for the four different Shroom proteins which have been characterised experimentally.

\begin{tabular}{|c|c|c|c|c|}
\hline Shroom ${ }^{a}$ & 1 & 2 & 3 & $4 a$ \\
\hline 1 & $100 / \mathrm{NA} / 100 / 100^{\mathrm{b}}$ & $11.7 / \mathrm{NA} / 32.9 / 37.7$ & $10.9 / \mathrm{NA} / 29.8 / 32.9$ & $9.5 / \mathrm{NA} / \mathrm{NA} / 35.3$ \\
\hline 2 & & $100 / 100 / 100 / 100$ & $25.4 / 67.5 / 44.6 / 68.2$ & $20.1 / 61.0 / \mathrm{NA} / 65.8$ \\
\hline 3 & & & $100 / 100 / 100 / 100$ & $15.9 / 63.6 / \mathrm{NA} / 61.6$ \\
\hline $4 a$ & & & & $100 / 100 / N A / 100$ \\
\hline
\end{tabular}

a This table makes use of the new nomenclature presented in Table I. To avoid evolution-based dissimilarity, the human homologues have been used in the analysis. b Percent sequence identity is given in the format global/PDZ/ASDI/ASD2; NA, not applicable. Global sequence identity is based on those residues aligning to hShroom I residues I - 826. The alignments on which this matrix is based were created using ClustalW. They are available upon request. 